\title{
Roles of Gremlin 1 and Gremlin 2 in regulating ovarian primordial to primary follicle transition
}

\author{
Eric E Nilsson, Ginger Larsen and Michael K Skinner \\ School of Biological Sciences, Center for Reproductive Biology, Washington State University, Pullman, \\ Washington 99164-4236, USA \\ Correspondence should be addressed to M K Skinner; Email: skinner@wsu.edu
}

\begin{abstract}
A network of extracellular signaling factors has previously been shown to act in concert to control the ovarian primordial to primary follicle transition. The current study was designed to investigate the roles of the endogenous bone morphogenetic protein (BMP) inhibitors Gremlin 1 (GREM1) and GREM2 in primordial follicle transition in the rat ovary. GREM1 and GREM2 treatments were found to reverse the effects of anti-Müllerian hormone (AMH) to inhibit follicle transition in a whole-ovary culture system. GREM1 reversed the effect of BMP4 to stimulate primordial follicle transition. Immunohistochemical studies showed that GREM2, but not GREM1, was present in primordial follicles suggesting that GREM2 may regulate primordial follicle transition in vivo. Co-immunoprecipitation studies indicated that GREM2 directly binds to AMH, as well as to BMP4. Transcriptome analyses of ovaries treated with GREM2 or GREM1 yielded negligible numbers of differentially expressed genes, suggesting that the immediate effects of GREM2 or GREM1 appear to be at the level of protein-protein interactions, rather than direct actions on the cells. A number of other ovarian growth factors were found to influence the expression of Grem2. Observations suggest that Grem2 is a part of the signaling network of growth factors that regulate the primordial to primary follicle transition. Insights into the regulatory networks affecting the pool of primordial follicles are important to understand the molecular basis for reproductive diseases such as primary ovarian insufficiency.
\end{abstract}

Reproduction (2014) 147 865-874

\section{Introduction}

In the current study, the effects of the bone morphogenetic protein (BMP) inhibitors Gremlin 1 (GREM1) and GREM2 on ovarian primordial follicle development are investigated. Oocytes are stored in an arrested state in mammalian ovaries in structures called primordial follicles. Each primordial follicle contains an oocyte that is arrested in prophase I of meiosis, surrounded by a single layer of flattened (squamous) pre-granulosa cells (Hirshfield 1991, Rajah et al. 1992). During reproductive life, a small number of primordial follicles continually leave the arrested state by undergoing the primordial to primary follicle transition. This transition involves an increase in oocyte size, the granulosa cells become cuboidal and proliferate, and recruitment of precursor theca cells occurs. Following follicle transition, the follicles either undergo atresia and regress, or ovulate the oocyte from a large antral follicle (Peters et al. 1975). When the pool of arrested primordial follicles becomes depleted, reproduction ceases and women enter menopause.

Control of the primordial to primary follicle transition is vitally important for successful reproduction. A network of growth factors and other extracellular signaling factors act in concert to create the correct balance for promoting or inhibiting primordial follicle transition (Pangas 2007, Nilsson et al. 2010). In this way, the available pool of primordial follicles is metered out so as to last for the duration of the expected reproductive lifespan (Pangas 2012). If this balance is upset, then infertility and other reproductive pathologies such as primary ovarian insufficiency (POI) can occur. In cases of $\mathrm{POI}$, the pool of primordial follicles is depleted early in life and women in their 30s or even 20s cease ovulating and enter menopause (Coulam et al. 1986, Maclaran \& Panay 2011).

Growth factors that promote the primordial to primary follicle transition include kit ligand (Kitl; Parrott \& Skinner 1999), fibroblast growth factor 2 (Fgf2; Nilsson et al. 2001, Nilsson \& Skinner 2004), neurotrophins (Dole et al. 2008, Dissen et al. 2009, Nilsson et al. 2009), the transforming growth factor (TGF) family members Bmp4 (Nilsson \& Skinner 2003), Bmp7 (Lee et al. 2001), and others (Skinner 2005, Knight \& Glister 2006, Fortune et al. 2010, Buratini \& Price 2011, Chaves et al. 2012). Growth factors that inhibit the primordial follicle transition include $\mathrm{CxCl12}$ (Holt et al. 2006) and the TGF $\beta$ family member anti-Müllerian hormone ( $A m h$; Durlinger et al. 1999, 2002, Nilsson et al. 2007). While 
many secreted growth factors have been tested experimentally for their ability to regulate primordial follicle transition, less work has been done to investigate the role played by endogenous inhibitors of secreted growth factors.

Grem1 and Grem2 (or protein related to DAN and cerberus (PRDC)) are members of the DAN family of BMP inhibitors (Avsian-Kretchmer \& Hsueh 2004, Kattamuri et al. 2012). These endogenously produced inhibitors have similar bioactivities and are known to bind to members of the BMP family of growth factors and prevent the growth factors from activating their receptors (Kosinski et al. 2007, Segditsas et al. 2008). Both Grem1 and Grem2 have been shown to be expressed in the ovary and to have roles in regulating later stages of follicle development (Pangas et al. 2004, Sudo et al. 2004, Myers et al. 2011). However, neither Grem 1 nor Grem2 are known to have roles in regulating the primordial to primary follicle transition.

The objective of the current study was to investigate whether Grem1 and Grem2 are part of the network of paracrine growth factors and inhibitors that regulate the primordial to primary follicle transition. Organ culture experiments using intact rat ovaries directly tested the effects of GREM1 and GREM2 proteins on primordial follicle transition. Immunohistochemistry was used to identify the follicle stages and cell types in the ovary that express GREM1 and GREM2. Co-immunoprecipitation experiments tested the ability of GREM2 to bind directly to BMP4 and $\mathrm{AMH}$. A greater basic understanding of how the network of paracrine signaling factors acts to regulate the recruitment of follicles from the arrested primordial follicle pool may help in understanding the causes of certain types of infertility in women, such as POI.

\section{Materials and methods \\ Animals and ovary culture}

In this experiment, 4-day-old female Sprague-Dawley rats (Harlan Laboratories, Inc., Indianapolis, IN, USA) were killed according to the Washington State University IACUC committee-approved protocol (IACUC \#02568) and their ovaries removed and cultured as a whole as described previously (Dole et al. 2008). Ovaries of 4-day-old rats contain primarily primordial follicles. Whole ovaries were cultured on floating filters (0.4 $\mu \mathrm{m}$ Millicell-CM, Millipore, Bedford, MD, USA) in $0.5 \mathrm{ml}$ DMEM-Ham's F-12 medium (1:1, vol/vol) containing $0.1 \%$ BSA (Sigma), 0.1\% Albumax (Gibco BRL), $27.5 \mu \mathrm{g} / \mathrm{ml}$ transferrin, and $0.05 \mathrm{mg} / \mathrm{ml}$ L-ascorbic acid (Sigma) in a fourwell culture plate (Nunc plate, Applied Scientific, South San Francisco, CA, USA) for 2 or 10 days. The medium was supplemented with penicillin (400 units/ml), streptomycin $(400 \mu \mathrm{g} / \mathrm{ml})$, and gentamycin $(100 \mu \mathrm{g} / \mathrm{ml})$ to prevent bacterial contamination. Ovaries were randomly assigned to treatment groups with one to three ovaries per floating filter per well. Wells were treated every 2 days with the following recombinant proteins alone or in combination: mouse GREM $(50 \mathrm{ng} / \mathrm{ml}$;
R\&D Systems, Minneapolis, MN, USA, cat no. 956-GR), mouse GREM2 (PRDC) (100 ng/ml; R\&D Systems, cat no. 2069$\mathrm{PR})$, human AMH (50 ng/ml; R\&D Systems, cat no. 1737-MS), mouse KITLG (50ng/ml; R\&D Systems, cat no. 455-MC), and rat FGF2 (50 ng/ml; R\&D Systems, cat no. 3339-FB). GREM1 and GREM2 dosages were chosen based on effective dosages for inhibiting BMP stimulatory activity in cell culture, as provided by the manufacturer. Preliminary studies using 4-day-old rat whole-ovary culture showed no difference in the effect of GREM1 on follicle development between dosages 50 and $150 \mathrm{ng} / \mathrm{ml}$. After 10 days of culture, ovaries were fixed in Bouin's fixative (Sigma) for $1 \mathrm{~h}$. Ovaries were then embedded in paraffin, sectioned at $3 \mu \mathrm{m}$, and stained with hematoxylin/eosin for use in morphological analysis. Ovaries from 4-day-old rats cultured for 10 days have follicles in pre-antral stages having up to three granulosa cell layers. Most follicles are morphologically normal (Supplementary Fig. 1, see section on supplementary data given at the end of this article).

\section{Morphological analysis}

For each ovary, the number of oocytes at each developmental stage was counted and the counts for each stage were averaged across the two consecutive histological sections that had the largest ovarian cross section. With two adjacent thin sections counted, it is expected that the sections are essentially equivalent, with the same follicles counted in most cases. If this is not the case, then these counts are discarded. The oocyte nucleus had to be visible for an oocyte to be counted. Normally, between 70 and 150 follicles were present in each ovarian cross section. From 5 to 12 ovaries were evaluated per treatment group. Oocytes were classified as primordial (stage 0 ) or as one of the developing pre-antral stages (stages 1-4) as described previously (Parrott \& Skinner 1999, Kezele \& Skinner 2003). Primordial follicles consist of an oocyte partially or completely surrounded by a single layer of squamous pre-granulosa cells. Follicles with only one cuboidal granulosa cell visible, the rest being squamous, were also classified as primordial (Gougeon \& Chainy 1987, Gougeon \& Busso 2000, Meredith et al. 2000, de Bruin et al. 2002). Developing (stages 1-4) follicles contain successively more cuboidal granulosa cells in layers around the oocyte (Parrott \& Skinner 1999, Nilsson et al. 2001). The proportion (i.e. percentage) of developing follicles out of total follicles per cross section was calculated for each ovary. Data were expressed and graphed as proportion of developing follicles relative to controls, with the control means set equal to 1.0. This normalization to controls accounts for variations between litters in response to treatment and for minor differences between individuals reading the slides in categorizing follicles into different developmental stages, as differences between treatment groups were consistent. One individual performed counts for GREM1 experiments. Three individuals performed counts for GREM2 experiments, with the three periodically all counting the same ovary and comparing results.

\section{RNA collection}

For ovary culture experiments in which ovarian RNA was collected, two to three ovaries per well were cultured for 2 days 
either untreated (controls) or were treated with GREM1 $(50 \mathrm{ng} / \mathrm{ml})$ or GREM2 $(100 \mathrm{ng} / \mathrm{ml})$. After 2 days of culture, there are no morphological differences between control and growth factor-treated ovaries (Nilsson et al. 2006). Therefore, measurements of whole-ovary gene expression will reflect differences in RNA transcription rather than differing proportions of cell types due to differential cell proliferation between treatments. RNA was isolated from whole rat ovaries after homogenization in $1 \mathrm{ml}$ TRIzol reagent (Sigma-Aldrich), according to the manufacturer's instructions. Two or three ovaries from the same culture well (from different rat pups out of the same litter) and receiving the same treatment were pooled and homogenized together. Homogenized samples were stored at $-70^{\circ} \mathrm{C}$ until the time of RNA isolation.

\section{Immunohistochemistry}

Rat ovaries were fixed in $10 \%$ neutral buffered formalin, paraffin embedded, and sectioned using standard procedures. Ovary sections were immunostained as described previously (Nilsson et al. 2002) for the presence of GREM1 or GREM2 using an antiGremlin primary antibody (Mouse Gremlin antibody; R\&D Systems, cat no. AF956) or anti-Grem2 primary antibody (human Grem2 H-58; Santa Cruz Biotechnology, cat no. sc135103). Briefly, sections were de-paraffinized, rehydrated through a graded ethanol series, boiled in $10 \mathrm{mM}$ sodium citrate buffer, washed with $0.1 \%$ Triton-X solution, and then blocked with $10 \%$ rabbit serum or $10 \%$ goat serum for anti-Gremlin or anti-Grem2 experiments respectively (normal rabbit serum or normal goat serum; Vector Laboratories, Inc., Burlingame, CA, USA) for $20 \mathrm{~min}$ prior to incubation with primary antibody for $12 \mathrm{~h}$. Anti-GREM1 and anti-GREM2 antibodies were used at a concentration of $200 \mathrm{ng} / \mathrm{ml}$ and non-specific IgG at a concentration of $200 \mathrm{ng} / \mathrm{ml}$. The sections were then washed with PBS and incubated with 1:400 diluted biotin-conjugated secondary antibodies for 45 min (Biotinylated anti-goat IgG (BA-5000) or Biotinylated anti-rabbit IgG (BA-1000); Vector laboratories, Inc.) and then again washed several times before applying streptavidin peroxidase (Histostain-Plus Streptavidin-Peroxidase; Invitrogen, cat no. 50-420Z). A DAB color reaction was performed following the manufacturer's instructions (Peroxidase Substrate Kit DAB; Vector laboratories, Inc., cat no. SK-4100) to localize sites of GREM and GREM2 in tissues.

\section{Microarray transcriptome analysis}

The mRNA processing and hybridization were performed at the Genomics Core Laboratory, Center for Reproductive Biology, Washington State University (Pullman, WA, USA) using standard Affymetrix reagents and protocol. Briefly, mRNA was transcribed into cDNA with random primers and cRNA was transcribed and from that single-stranded sense DNA was synthesized which was fragmented and labeled with biotin. Biotin-labeled fragmented ssDNA was then hybridized to the Rat Gene 1.0 ST microarrays containing more than 27000 transcripts (Affymetrix, Santa Clara, CA, USA). Hybridized chips were scanned on Affymetrix Scanner 3000. CEL files containing raw data were then pre-processed and analyzed with Partek Genomic Suite 6.5 beta Software (Partek
Incorporated, St Louis, MO, USA) using an RMA (robust multi array averaging) and GC (guanine/cytosine)-content-adjusted algorithm. The signals from an average of 28 different probes for each transcript were compared to give a single value. Lists of differentially expressed genes for each treatment were generated using the following cutoff criteria: signal ratio of control/treatment $>1.20$ change, mean difference for un-logged signals between control and treatment $>10$, $t$-test $P$ values $<0.05$, and correcting for organ culture date batch effects. CEL files (MIAME compliant raw data) from this study have been deposited with the $\mathrm{NCBI}$ gene expression and hybridization array data repository (GEO, http://www.ncbi. nlm.nih.gov/geo, \#GSE pending) and can also be accessed through www.skinner.wsu.edu.

Previous studies have demonstrated that microarray data are validated with quantitative PCR data (Shima et al. 2004, Kezele et al. 2005). Owing to the presence of an average of 28 different oligonucleotide probes for each specific gene being used on the microarray, vs only a single primer set for a gene in quantitative PCR, the microarray is more effective at eliminating false positive and provides a more robust quantification of changes in gene expression.

\section{Co-immunoprecipitation}

Co-immunoprecipitation experiments were performed to determine which growth factors would bind to GREM2. Recombinant proteins GREM2, BMP4 (R\&D Systems, cat no. 314-BP), and AMH were placed alone or in combination in $1 \mathrm{ml}$ binding buffer $(0.5 \%$ BSA in PBS) for $1 \mathrm{~h}$ at room temperature with rocking. Pull-down antibodies were added to each reaction tube (anti-GREM2 (1000 ng/ml; Santa Cruz Biotechnology, cat no. sc-135103), anti-BMP4 (500 ng/ml; R\&D Systems, cat no. MAB757), or anti-AMH (1000 ng/ml; Santa Cruz Biotechnology, cat no. sc-34833)) and incubated at $4{ }^{\circ} \mathrm{C}$ overnight with rocking. Antibody-bound protein complexes were removed from solution by adding $20 \mu \mathrm{l}$ Protein A/G Agarose beads (pre-blocked with binding buffer) (Santa Cruz Biotechnology, cat no. sc-2003) to each reaction tube and incubating with rocking for $1 \mathrm{~h}$, then centrifuging for $5 \mathrm{~min}$. at $1000 \mathrm{~g}$ and discarding the supernatant. Beads were rinsed four times with blocking buffer, then re-suspended in $40 \mu \mathrm{l}$ SDS sample buffer for western blotting, and boiled to remove proteins from beads. Reaction samples were stored at $-20^{\circ} \mathrm{C}$ until the time of western blot.

Western blots were performed in duplicate to qualitatively evaluate growth factor binding using 15\% acrylamide/ $0.4 \%$ bis-acrylamide resolving gels and $5 \%$ acrylamide $/ 0.17 \%$ bis-acrylamide stacking gels, and then nitrocellulose transfer membranes. GREM2, BMP4, and AMH proteins were loaded as positive controls. Primary antibodies were anti-GREM2 (400 ng/ml; Santa Cruz Biotechnology, cat no. sc-135103), anti-BMP4 (5000 ng/ml; R\&D Systems, Minneapolis, MN, USA, cat no. MAB757), or anti-AMH $(2000 \mathrm{ng} / \mathrm{ml}$; Santa Cruz Biotechnology, cat no. sc-34883). Secondary HRP-conjugated antibodies were anti-rabbit IgG-HRP to detect anti-GREM2 antibody $(200 \mathrm{ng} / \mathrm{ml}$; Santa Cruz Biotechnology, cat no. sc-2004), anti-mouse IgG-HRP to detect anti-BMP4 antibody (1:1000 dilution; Cell Signaling Technology, Danvers, MA, USA, cat no. 7076), anti-goat IgG-HRP to detect anti-AMH antibody 
(1:1000 dilution; R\&D Systems, cat no. HAF017). Blots were visualized on film using the SuperSignal West Pico Chemiluminescent Substrate kit (Thermo Scientific, Pierce Biotechnology, Rockford, IL, USA, cat no. 34077).

\section{Statistical analysis}

Treatment groups are compared using ANOVA followed by post-hoc tests where appropriate. The data for GREM1 cultures and GREM2 cultures are both normally distributed, and thus parametric ANOVA is appropriate. The GREM2 treatment groups have unequal variances (AMH is small), and hence this must be taken into consideration. For GREM1, it was decided beforehand that our comparisons of interest were between untreated control vs GREM1, control vs BMP4, control vs $\mathrm{AMH}, \mathrm{BMP} 4$ vs GREM1 + BMP4, and AMH vs GREM1 + $\mathrm{AMH}$. Results of both Dunnett's and conservative Bonferroni's tests are now presented. For GREM2, it was similarly decided beforehand that our comparisons of interest were between untreated control vs GREM2, control vs $A M H$, and $A M H$ vs GREM2 $+\mathrm{AMH}$. As the variance for the AMH-treated group is small in GREM2 experiments, but similar to that of other treatments in the GREM1 experiments, one might conclude that the population variance for $\mathrm{AMH}$ is normal, and thus regular ANOVA and post-tests would be appropriate for GREM2 studies. However, this may not be the case, and hence the results of alternate analyses appropriate for unequal variances (the Kruskal-Wallis test, Dunn's post-hoc test, and Student's t-test with Welch's correction) are also presented. Groups were considered as statistically significant with $P \leq 0.05$. Statistics were calculated using GraphPad Prism, version 5.0b for Macintosh, GraphPad Software (San Diego, CA, USA).

\section{Results}

Ovaries were isolated from 4-day-old rats for use in organ culture experiments to test the effects of GREM1 and GREM2 on the primordial to primary follicle transition. Ovaries of 4-day-old rats contain predominantly primordial follicles. Whole ovaries were cultured intact for 10 days as described in 'Materials and methods' section in the presence or absence of GREM1, GREM2, BMP4, or AMH. Following culture, ovarian sections were obtained and evaluated morphologically to determine the numbers of primordial and developing follicles per section (Figs 1 and 2).

Treatment of ovaries with GREM1 alone did not result in any change in the proportion of developing follicles compared with controls (Fig. 1). Using both Dunnett's and conservative Bonferroni's post-hoc tests, treatment with BMP4 increased the proportion of developing follicles, indicating an increase in the primordial to primary follicle transition. AMH treatment resulted in a decrease in developing follicles as expected. Co-treatment of ovaries with both GREM1 and BMP4 reversed the effects of BMP4. Similarly, co-treatment with both GREM1 and $\mathrm{AMH}$ reversed the effects of $\mathrm{AMH}$ on follicle

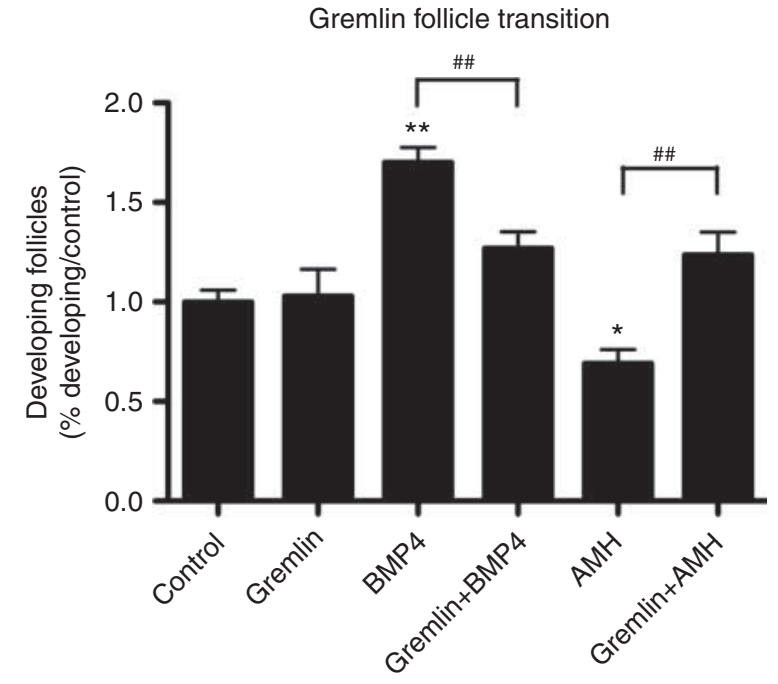

Figure 1 Primordial to primary follicle transition after GREM1 treatment. The follicles per ovarian cross section were counted and categorized as either primordial or developing (i.e. after primordial to primary follicle transition) after organ culture. Data are expressed as the proportion of developing follicles for each treatment divided by the proportion of developing follicles in the control group. A total of 5-12 ovaries were evaluated per treatment group, for six experiments performed in replicate. ${ }^{*} P<0.05$ and ${ }^{* *} P<0.01$ compared with control by Dunnett's post-hoc test after significant ANOVA result. ${ }^{\#} P<0.005$ by Student's $t$-test.

transition. These results indicate that GREM1 is inhibitory to the actions of BMP4 and also AMH.

Although the biological activities of GREM1 and GREM2 have been shown to be similar (Kosinski et al. 2007, Segditsas et al. 2008), experiments were performed in which cultured ovaries were treated with GREM2 and/or AMH. Both Dunnett's and non-parametric Dunn's post-hoc tests showed GREM2- and AMHtreated groups to have a decrease in developing follicles compared with the control. By contrast, the conservative Bonferroni's test did not identify any groups as significantly different from each other. However, a $t$-test using Welch's correction for unequal variances showed a significant difference between the AMH-treated group and the group co-treated with AMH and GREM2. Both parametric (ANOVA) and non-parametric (KruskalWallis) analyses were performed. Although the data for GREM2 culture experiments are normally distributed, the possibility of unequal variances between these treatment groups must be taken into consideration. Together, these data suggest that treatment of ovaries with GREM2 resulted in a modest but statistically significant decrease in developing follicles (Fig. 2). A preliminary organ culture experiment demonstrated that GREM2 inhibited BMP4 actions similar to GREM1 (data not shown) as would be expected from the previous literature (Kosinski et al. 2007, Kattamuri et al. 2012). As expected, treatment with $\mathrm{AMH}$ resulted in a decrease in developing follicles. Interestingly, co-treatment with 


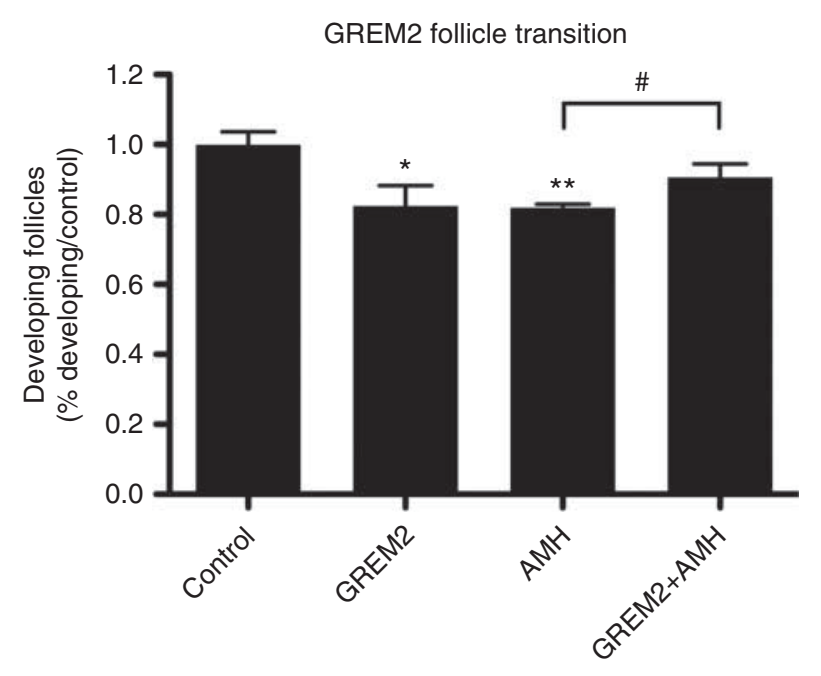

Figure 2 Primordial to primary follicle transition after GREM2 treatment. The follicles per ovarian cross section were counted and categorized as either primordial or developing (i.e. after primordial to primary follicle transition) after organ culture. Data are expressed as the proportion of developing follicles for each treatment divided by the proportion of developing follicles in the control group. A total of 6-11 ovaries were evaluated per treatment group, for five experiments performed in replicate. ${ }^{*} P<0.05$ and ${ }^{* *} P<0.01$ compared with control by Dunnett's post-hoc test after significant ANOVA test result. ${ }^{*} P \leq 0.05$ by Student's t-test with Welch's correction for unequal variances.

both GREM2 and $A M H$ reversed the effect of $A M H$ treatment. These results suggest that GREM2 is inhibitory to the actions of $\mathrm{AMH}$.

Immunohistochemical experiments were performed to localize the expression of GREM1 and GREM2 in rat ovaries (Figs 3 and 4). GREM1 protein is detectable in the granulosa cells and oocytes of large developing follicles (Fig. 3). However, expression of GREM1 in primordial follicles was not detected. This suggests that GREM1 may not participate in primordial follicle transition in vivo. GREM2 protein is present in the granulosa cells of both developing and primordial follicles (Fig. 4). GREM2 also appears to be present in the nuclei of some oocytes. Therefore, GREM2 is expressed in primordial follicles, while both GREM2 and GREM1 are expressed in laterstage developing follicles having several layers of granulosa cells.

As co-treatment of ovaries with both GREM2 and $\mathrm{AMH}$ reversed the effects of $\mathrm{AMH}$ on the primordial to primary follicle transition, co-immunoprecipitation experiments were performed to test whether GREM2 was bound to $\mathrm{AMH}$ and inhibited its action directly. Qualitative western blots were used to evaluate co-immunoprecipitation and growth factor binding. Successful immunoprecipitation and western blot detection of GREM2 (lane 5), AMH (lane 10), and BMP4 (lane 14) were demonstrated. Control experiments were performed to show that GREM2 was bound to its known binding partner BMP4 (Fig. 5). When GREM2 and BMP4 are incubated together, precipitation with anti-BMP4 antibody also co-precipitates GREM2 allowing detection of GREM2 by western blot (Fig. 5, lane 1). Similarly, incubation of GREM2 with BMP4, followed by precipitation with anti-GREM2 antibody, results in detection of BMP4 (Fig. 5, lane 12). When a negative control was performed where GREM2 and BMP4 are

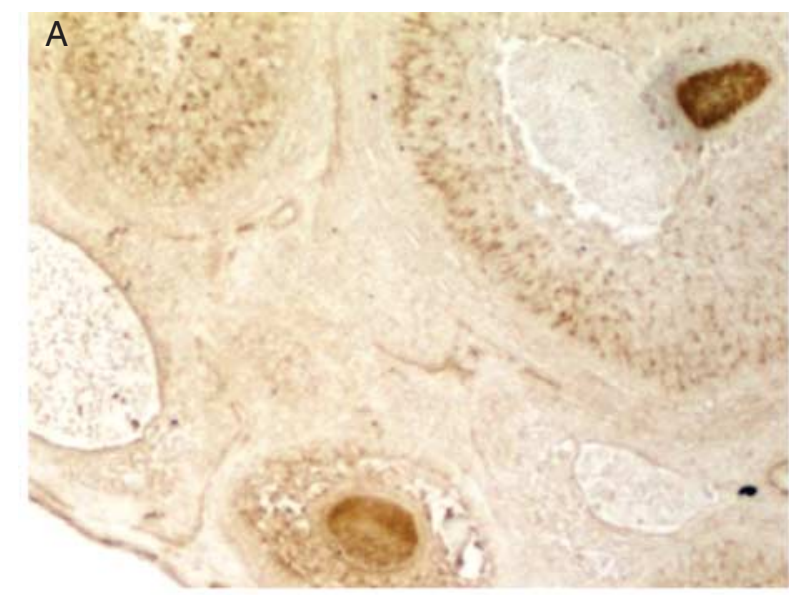

B

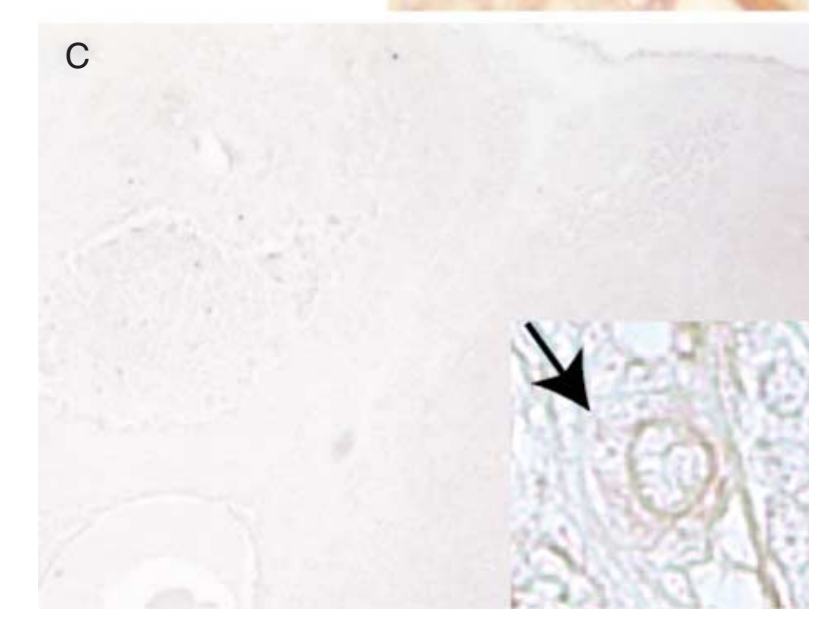

Figure 3 Ovarian immunohistochemical localization of GREM1 in 19-day-old ovaries. (A) GREM1 is expressed in the granulosa cells of developing follicles with several layers of granulosa cells. (B) Higher magnification image showing primordial follicles (arrows) with no distinct GREM1 expression. (C) Negative control using non-specific IgG as a primary antibody and inset at a higher magnification with an arrow indicating a primordial follicle. 


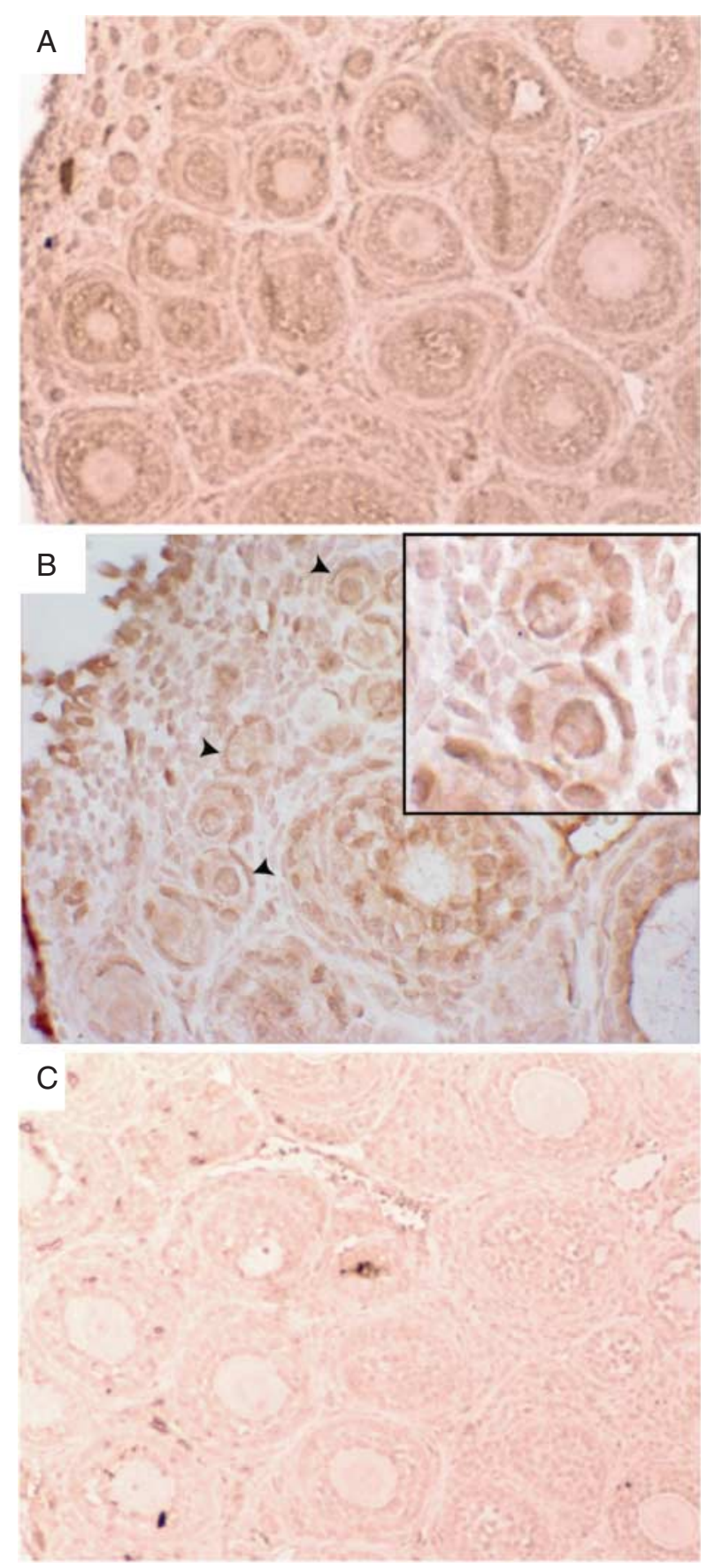

Figure 4 Ovarian immunohistochemical localization of GREM2 in 14-day-old ovaries. (A) GREM2 is prominently expressed in the granulosa cells of developing follicles with several layers of granulosa cells. (B) Higher magnification image showing GREM2 expression in the flattened granulosa cells (arrowheads) of primordial follicles. (Inset) Higher magnification view showing GREM2 expression in a primordial (lower) and transitional follicle. (C) Negative control using non-specific IgG as a primary antibody.

incubated together with no precipitating antibody, neither GREM2 nor BMP4 is detected by western blot (Fig. 5, lanes 3 and 13). The anti-BMP4 antibody does not bind and precipitate GREM2 (Fig. 5, lane 6).
The anti-GREM2 antibody does bind and precipitate a small amount of BMP4, but this small band is less than the BMP4 band observed when BMP4 co-precipitates with GREM2 using the anti-GREM2 antibody (Fig. 5, lane 15 vs lane 12). Observations indicate that GREM2 binds to BMP4 as expected.

When GREM2 and $\mathrm{AMH}$ are incubated together, precipitation with anti-AMH antibody also co-precipitates GREM2 (Fig. 5, lane 2). Similarly, incubation of GREM2 with $\mathrm{AMH}$, followed by precipitation with anti-GREM2 antibody, results in detection of AMH (Fig. 5, lane 8). This suggests that GREM2 binds to AMH. When GREM2 and $\mathrm{AMH}$ are co-incubated with no precipitating antibody, no GREM2 is detected by western blot (Fig. 5, lane 4), but some AMH is detectable (Fig. 5, lane 9). However, there was less AMH than detected when GREM2 and AMH were incubated together followed by co-precipitation with anti-GREM2 antibody (Fig. 5, lane 8 vs lane 9). The anti-AMH antibody does not non-specifically bind to GREM2 (Fig. 5, lane 7). The anti-GREM2 antibody may bind to a trace amount of AMH (Fig. 5, lane 11), or this faint band may reflect a small amount of non-specific AMH protein carry-over to the western blot, similar to lane 9. In summary, GREM2 and AMH are found to bind when precipitated by anti-AMH antibody. When GREM2-AMH complexes

A
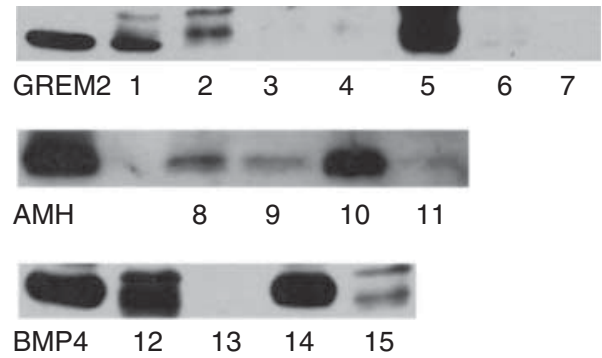

B

\begin{tabular}{|l|l|l|l|}
\hline Lane & $\begin{array}{c}\text { Co-immunoprecipitation } \\
\text { proteins }\end{array}$ & Precipitating Ab & $\begin{array}{r}\text { Western detection } \\
\text { Ab against }\end{array}$ \\
\hline 1 & GREM2+BMP4 & Anti-BMP4 & GREM2 \\
\hline 2 & GREM2+AMH & Anti-AMH & GREM2 \\
\hline 3 & GREM2+BMP4 & None & GREM2 \\
\hline 4 & GREM2+AMH & None & GREM2 \\
\hline 5 & GREM2 & Anti-GREM2 & GREM2 \\
\hline 6 & GREM2 & Anti-BMP4 & GREM2 \\
\hline 7 & GREM2 & Anti-AMH & GREM2 \\
\hline 8 & GREM2+AMH & Anti-GREM2 & AMH \\
\hline 9 & GREM2+AMH & None & AMH \\
\hline 10 & AMH & Anti-AMH & AMH \\
\hline 11 & AMH & Anti-GREM2 & AMH \\
\hline 12 & GREM2+BMP4 & Anti-GREM2 & BMP4 \\
\hline 13 & GREM2+BMP4 & None & BMP4 \\
\hline 14 & BMP4 & Anti-BMP4 & BMP4 \\
\hline 15 & BMP4 & Anti-GREM2 & BMP4 \\
\hline
\end{tabular}

Figure 5 GREM2 co-immunoprecipitation. (A) Western blots of samples after co-immunoprecipitation detecting GREM2, AMH, and BMP4.

(B) Lane numbers describing the proteins combined, the antibody used for co-immunoprecipitation, and the antibody used for protein detection in the western blot for each sample. 
are precipitated by anti-GREM2 antibody, GREM2-AMH binding is confirmed by the results obtained. Observations suggest that GREM2 binds to AMH. Co-immunoprecipitation experiments to test whether GREM1 was bound to $\mathrm{AMH}$ were attempted, but issues of crossreactivity of antibodies to these proteins rendered the results uninterpretable (data not shown).

In order to investigate the effects that GREM2 and GREM 1 have on gene expression in the ovary, RNA was collected from whole cultured ovaries from 4-day-old rats treated for 2 days with GREM2, GREM1, or left untreated as controls. A 2-day culture was used to reduce the confounding factor of morphological changes that are observed in the ovary after a 10-day culture. RNA was subjected to microarray analysis using Affymetrix Rat Gene 1.0 ST arrays (see 'Materials and methods' section). GREM2 treatment resulted in identification of 57 differentially expressed transcripts compared with controls, of which 32 were annotated as genes. GREM1 treatment resulted in identification of 29 differentially expressed transcripts compared with controls, of which eight were annotated as genes (Supplementary Table 1, see section on supplementary data given at the end of this article). These differentially expressed genes were distributed among several functional categories, with no functions being obviously over-represented. With the exception of a couple of EST (Expressed Sequence Tags), the altered expression was $>1.2$ - and $<1.5$-fold change (increases and decreases), such that negligible effects on gene expression were observed (Supplementary Table 1).

Microarray data from a previous study (Nilsson et al. 2010) were interrogated to further examine the expression of Grem 1 and Grem2 during early follicle development. In this study of the network of genes regulating primordial follicle transition, ovaries from 4-day-old rats were treated with several different growth factors for 2 days and then RNA was collected for microarray analysis (Nilsson et al. 2010). The relative

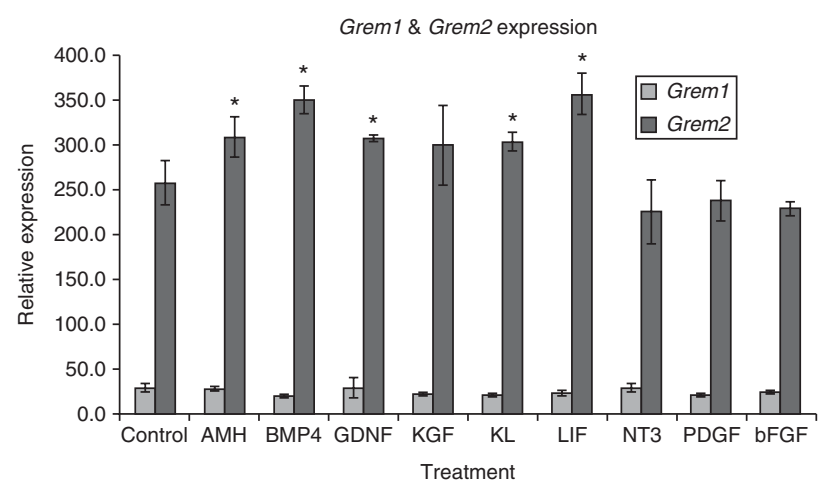

Figure 6 Expression of Grem1 and Grem2 mRNA in 4-day-old rat ovaries cultured for 2 days with the growth factor treatments indicated. Expression of mRNA is shown as measured by microarray analysis. *Expression significantly $(P<0.05)$ different from untreated controls by Student's $t$-test. Modified from Nilsson et al. (2010). expression of Grem1 and Grem2 in response to the different growth factor treatments is shown in Fig. 6 . The expression levels of Grem 1 were approximately the background levels and the detection limit of the microarray, while expression levels of Grem2 were well above the background levels. This suggests that Grem1 mRNA expression is quite low when compared with Grem2 mRNA expression. As 4-day-old ovaries cultured for 2 days contain only primordial and early-stage follicles, this is consistent with only Grem2 acting to regulate the primordial to primary follicle transition. It can also be observed that treatment with these growth factors that are known to regulate the primordial follicle transition, including $\mathrm{AMH}$ and $\mathrm{BMP} 4$, often results in significant increases in Grem2 expression compared with untreated controls. This further suggests that Grem2 is a part of the network of growth factor signaling controlling early follicle development, perhaps playing an autoregulatory feedback role to prevent excessive signaling (Glister et al. 2011).

\section{Discussion}

The roles that Grem1 and Grem2 might play in regulating the primordial to primary follicle transition were investigated using the rat as a model. Ovary organ culture experiments showed that GREM1 was able to reverse the stimulatory effect that BMP4 had on primordial follicle transition (Fig. 1). This is because GREM1 is known to bind and inhibit the actions of BMPs (Avsian-Kretchmer \& Hsueh 2004, Rosen 2006, Kattamuri et al. 2012). Interestingly, GREM1 treatment was also able to reverse the inhibitory effect that $\mathrm{AMH}$ had on primordial follicle transition. This raises the possibility that GREM1 is able to directly bind and inhibit the actions of $\mathrm{AMH}$, which like BMPs is a member of the TGF $\beta$ family of proteins.

GREM2 was also able to reverse the inhibitory effect that $\mathrm{AMH}$ had on primordial follicle transition, similar to GREM1 (Fig. 2). GREM2 treatment alone had an inhibitory effect on follicle transition, but interestingly this effect was not additive with that of $\mathrm{AMH}$. Rather, GREM2 reversed the effect of $\mathrm{AMH}$. One potential explanation for the inhibitory effect observed when ovaries were treated with GREM2 alone is that GREM2 binds and inhibits the actions of BMPs that are produced endogenously in the cultured ovaries. Preliminary organ culture experiments supported the ability of GREM2 to inhibit BMP4 stimulation of follicle transition (data not shown), as would be expected from previous literature (Avsian-Kretchmer \& Hsueh 2004, Kosinski et al. 2007, Kattamuri et al. 2012). BMP4 and BMP7 are known stimulators of primordial follicle transition (Lee et al. 2001, Nilsson \& Skinner 2003), thus the well-established function of GREM2 to bind to BMPs (Kattamuri et al. 2012) could result in a suppression of follicle transition. However, if GREM2 also directly binds and inhibits the 
actions of $\mathrm{AMH}$, then for those ovaries treated with both GREM2 and $\mathrm{AMH}$, the exogenous AMH could occupy the available GREM2 and release the endogenous BMPs preventing them from inhibition. Alternatively, GREM2 treatment may change signaling cascades in other ways that result in a net decrease in follicle transition. Further studies will be required to determine the exact mechanism by which GREM2 treatment alone decreases the primordial to primary follicle transition.

Immunohistochemical experiments showed that both GREM1 and GREM2 proteins are present in the granulosa cells of later-stage developing follicles that have multiple layers of granulosa cells (Figs 3 and 4). These results are in agreement with the findings from previous in situ hybridization studies that showed Grem1 and Grem2 expression to be localized to the granulosa cells of developing follicles (Pangas et al. 2004, Sudo et al. 2004). In a study by Fenwick et al. (2011), Grem2 mRNA was detected at much higher levels than Grem 1 mRNA in preantral follicles by PCR. This is consistent with the mRNA expression results of Nilsson et al. (2010) (Fig. 6) that again suggest that in ovaries containing only primordial and preantral stage follicles mRNA expression of Grem2 is higher than that of Grem1. Neither Grem1 nor Grem2 mRNA was detected in mouse oocytes (Fenwick et al. 2011), while in the current study GREM proteins were detectable in some oocytes. This might reflect species differences or paracrine regulation. In the current study, GREM2, but not GREM1, was also detected in primordial follicles. This suggests that while either GREM1 or GREM2 can affect the regulation of the primordial follicle transition in an experimental organ culture system, only GREM2 is present locally to act on primordial follicles in vivo. This does not exclude the possibility that GREM1 from later-stage developing follicles acts on distant primordial follicles, but such later-stage follicles are not present in the neonatal ovaries used in these experiments.

As GREM2 was shown to reverse the effects of $\mathrm{AMH}$ on the primordial to primary follicle transition, co-immunoprecipitation experiments were performed to test if GREM2 binds directly to $\mathrm{AMH}$, as well as to its known binding partner BMP4 (Kattamuri et al. 2012). Combined results indicate that GREM2 binds to $A M H$, as well as BMP4 (Fig. 5). This explains how GREM2 treatment alone can inhibit the primordial to primary follicle transition in cultured ovaries, and yet GREM2 treatment in combination with $\mathrm{AMH}$ will reverse the inhibitory effect of $\mathrm{AMH}$. Further studies are needed to successfully investigate if GREM1 will also directly bind and inhibit the actions of $\mathrm{AMH}$.

Microarray analyses of mRNA isolated from ovaries treated with GREM2 or GREM1 yielded negligible numbers of differentially expressed genes compared with controls with a 1.2- to 1.5-fold change in expression for all except several ESTs, and no annotated genes $>1.5$-fold change (Supplementary Table 1).

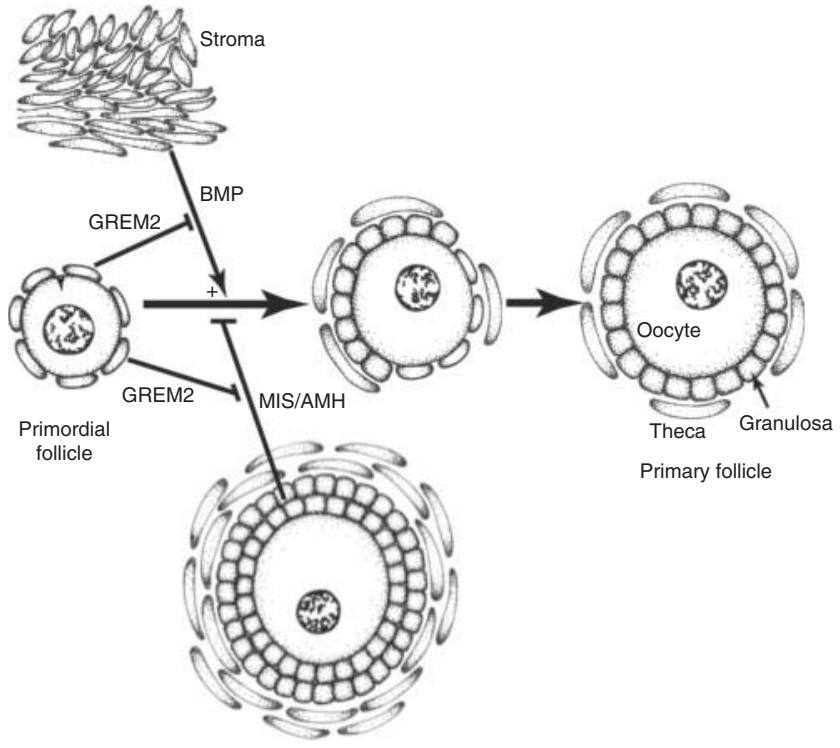

Figure 7 Model of GREM2 actions affecting the primordial to primary follicle transition. GREM2 is secreted by granulosa cells of primordial and developing follicles. GREM2 acts to bind and inhibit the actions of $\mathrm{BMP} 4$ from stroma and $\mathrm{AMH}$ from granulosa of developing follicles.

Furthermore, no particular physiological processes or functional categories were identified as being overrepresented among these differentially expressed genes. This suggests that the immediate effects of GREM2 (or GREM1) appear to be at the level of protein-protein interactions rather than by induction of changes in gene expression.

In summary, these results show that Grem2 is a part of the signaling network of growth factors and other genes that regulate the primordial to primary follicle transition (Fig. 7). While both GREM1 and GREM2 are able to affect follicle transition in cultured ovaries, only GREM2 is normally present in primordial and early-stage developing follicles. GREM2 acts as an inhibitor of $\mathrm{BMPs}$ and $\mathrm{AMH}$ through direct protein-protein interactions (Fig. 7). An improved understanding of the regulatory network controlling the primordial to primary follicle transition is important for understanding the basis of reproductive diseases affecting the pool of primordial follicles, such as POI.

\section{Supplementary data}

This is linked to the online version of the paper at http://dx.doi. org/10.1530/REP-14-0005.

\section{Declaration of interest}

The authors declare that there is no conflict of interest that could be perceived as prejudicing the impartiality of the research reported. 


\section{Funding}

This work was supported by an NIH grant to M K Skinner.

\section{Acknowledgements}

We thank Ms Heather Johnson for assistance in preparation of the manuscript.

\section{References}

Avsian-Kretchmer O \& Hsueh AJ 2004 Comparative genomic analysis of the eight-membered ring cystine knot-containing bone morphogenetic protein antagonists. Molecular Endocrinology 18 1-12. (doi:10.1210/ me.2003-0227)

de Bruin JP, Dorland M, Spek ER, Posthuma G, van Haaften M, Looman CW \& te Velde ER 2002 Ultrastructure of the resting ovarian follicle pool in healthy young women. Biology of Reproduction 66 1151-1160. (doi:10.1095/biolreprod66.4.1151)

Buratini J \& Price CA 2011 Follicular somatic cell factors and follicle development. Reproduction, Fertility, and Development 23 32-39. (doi:10.1071/RD10224)

Chaves RN, de Matos MH, Buratini J Jr \& de Figueiredo JR 2012 The fibroblast growth factor family: involvement in the regulation of folliculogenesis. Reproduction, Fertility, and Development 24 905-915. (doi:10.1071/RD11318)

Coulam CB, Adamson SC \& Annegers JF 1986 Incidence of premature ovarian failure. Obstetrics and Gynecology 67 604-606.

Dissen GA, Garcia-Rudaz C \& Ojeda SR 2009 Role of neurotrophic factors in early ovarian development. Seminars in Reproductive Medicine 27 24-31. (doi:10.1055/s-0028-1108007)

Dole G, Nilsson EE \& Skinner MK 2008 Glial-derived neurotrophic factor promotes ovarian primordial follicle development and cell-cell interactions during folliculogenesis. Reproduction 135 671-682. (doi:10.1530/REP-07-0405)

Durlinger AL, Kramer P, Karels B, de Jong FH, Uilenbroek JT, Grootegoed JA \& Themmen AP 1999 Control of primordial follicle recruitment by anti-Müllerian hormone in the mouse ovary. Endocrinology 140 5789-5796.

Durlinger AL, Gruijters MJ, Kramer P, Karels B, Ingraham HA, Nachtigal MW, Uilenbroek JT, Grootegoed JA \& Themmen AP 2002 Anti-Müllerian hormone inhibits initiation of primordial follicle growth in the mouse ovary. Endocrinology 143 1076-1084.

Fenwick MA, Mansour YT, Franks S \& Hardy K 2011 Identification and regulation of bone morphogenetic protein antagonists associated with preantral follicle development in the ovary. Endocrinology 152 3515-3526. (doi:10.1210/en.2011-0229)

Fortune JE, Yang MY \& Muruvi W 2010 The earliest stages of follicular development: follicle formation and activation. Society of Reproduction and Fertility Supplement 67 203-216.

Glister C, Satchell L \& Knight PG 2011 Granulosal and thecal expression of bone morphogenetic protein- and activin-binding protein mRNA transcripts during bovine follicle development and factors modulating their expression in vitro. Reproduction 142 581-591. (doi:10.1530/REP-11-0150)

Gougeon A \& Busso D 2000 Morphologic and functional determinants of primordial and primary follicles in the monkey ovary. Molecular and Cellular Endocrinology 163 33-41. (doi:10.1016/S03037207(00)00220-3)

Gougeon A \& Chainy GB 1987 Morphometric studies of small follicles in ovaries of women at different ages. Journal of Reproduction and Fertility 81 433-442. (doi:10.1530/jrf.0.0810433)

Hirshfield AN 1991 Development of follicles in the mammalian ovary. International Review of Cytology 124 43-101.

Holt JE, Jackson A, Roman SD, Aitken RJ, Koopman P \& McLaughlin EA 2006 CXCR4/SDF1 interaction inhibits the primordial to primary follicle transition in the neonatal mouse ovary. Developmental Biology 293 449-460. (doi:10.1016/j.ydbio.2006.02.012)

Kattamuri C, Luedeke DM, Nolan K, Rankin SA, Greis KD, Zorn AM \& Thompson TB 2012 Members of the DAN family are BMPantagonists that form highly stable noncovalent dimers. Journal of Molecular Biology 424 313-327. (doi:10.1016/j.jmb.2012.10.003)
Kezele P \& Skinner MK 2003 Regulation of ovarian primordial follicle assembly and development by estrogen and progesterone: endocrine model of follicle assembly. Endocrinology 144 3329-3337. (doi:10.1210/en.2002-0131)

Kezele PR, Ague JM, Nilsson E \& Skinner MK 2005 Alterations in the ovarian transcriptome during primordial follicle assembly and development. Biology of Reproduction 72 241-255. (doi:10.1095/biolreprod. 104.032060)

Knight PG \& Glister C 2006 TGF- $\beta$ superfamily members and ovarian follicle development. Reproduction 132 191-206. (doi:10.1530/rep. 1.01074)

Kosinski C, Li VS, Chan AS, Zhang J, Ho C, Tsui WY, Chan TL, Mifflin RC, Powell DW, Yuen ST et al. 2007 Gene expression patterns of human colon tops and basal crypts and BMP antagonists as intestinal stem cell niche factors. PNAS 104 15418-15423. (doi:10.1073/pnas. 0707210104)

Lee WS, Otsuka F, Moore RK \& Shimasaki S 2001 Effect of bone morphogenetic protein-7 on folliculogenesis and ovulation in the rat. Biology of Reproduction 65 994-999. (doi:10.1095/biolreprod65.4.994)

Maclaran K \& Panay N 2011 Premature ovarian failure. Journal of Family Planning and Reproductive Health Care 37 35-42. (doi:10.1136/jfprhc. 2010.0015)

Meredith S, Dudenhoeffer G \& Jackson K 2000 Classification of small type $\mathrm{B} / \mathrm{C}$ follicles as primordial follicles in mature rats. Journal of Reproduction and Fertility 119 43-48.

Myers M, Tripurani SK, Middlebrook B, Economides AN, Canalis E \& Pangas SA 2011 Loss of gremlin delays primordial follicle assembly but does not affect female fertility in mice. Biology of Reproduction $\mathbf{8 5}$ 1175-1182. (doi:10.1095/biolreprod.111.091728)

Nilsson EE \& Skinner MK 2003 Bone morphogenetic protein-4 acts as an ovarian follicle survival factor and promotes primordial follicle development. Biology of Reproduction 69 1265-1272. (doi:10.1095/ biolreprod.103.018671)

Nilsson EE \& Skinner MK 2004 Kit ligand and basic fibroblast growth factor interactions in the induction of ovarian primordial to primary follicle transition. Molecular and Cellular Endocrinology 214 19-25. (doi:10. 1016/j.mce.2003.12.001)

Nilsson E, Parrott JA \& Skinner MK 2001 Basic fibroblast growth factor induces primordial follicle development and initiates folliculogenesis. Molecular and Cellular Endocrinology 175 123-130. (doi:10.1016/ S0303-7207(01)00391-4)

Nilsson EE, Kezele P \& Skinner MK 2002 Leukemia inhibitory factor (LIF) promotes the primordial to primary follicle transition in rat ovaries. Molecular and Cellular Endocrinology 188 65-73. (doi:10.1016/S03037207(01)00746-8)

Nilsson EE, Detzel C \& Skinner MK 2006 Platelet-derived growth factor modulates the primordial to primary follicle transition. Reproduction 131 1007-1015. (doi:10.1530/rep.1.00978)

Nilsson E, Rogers N \& Skinner MK 2007 Actions of anti-Müllerian hormone on the ovarian transcriptome to inhibit primordial to primary follicle transition. Reproduction 134 209-221. (doi:10.1530/REP-07-0119)

Nilsson E, Dole G \& Skinner MK 2009 Neurotrophin NT3 promotes ovarian primordial to primary follicle transition. Reproduction 138 697-707. (doi:10.1530/REP-09-0179)

Nilsson EE, Savenkova MI, Schindler R, Zhang B, Schadt EE \& Skinner MK 2010 Gene bionetwork analysis of ovarian primordial follicle development. PLoS ONE 5 e11637. (doi:10.1371/journal.pone.0011637)

Pangas SA 2007 Growth factors in ovarian development. Seminars in Reproductive Medicine 25 225-234. (doi:10.1055/s-2007-980216)

Pangas SA 2012 Regulation of the ovarian reserve by members of the transforming growth factor $\beta$ family. Molecular Reproduction and Development 79 666-679. (doi:10.1002/mrd.22076)

Pangas SA, Jorgez CJ \& Matzuk MM 2004 Growth differentiation factor 9 regulates expression of the bone morphogenetic protein antagonist gremlin. Journal of Biological Chemistry 279 32281-32286. (doi:10. 1074/jbc.M403212200)

Parrott JA \& Skinner MK 1999 Kit-ligand/stem cell factor induces primordial follicle development and initiates folliculogenesis. Endocrinology $\mathbf{1 4 0}$ 4262-4271.

Peters H, Byskov AG, Himelstein-Braw R \& Faber M 1975 Follicular growth: the basic event in the mouse and human ovary. Journal of Reproduction and Fertility 45 559-566. (doi:10.1530/jrf.0.0450559) 
Rajah R, Glaser EM \& Hirshfield AN 1992 The changing architecture of the neonatal rat ovary during histogenesis. Developmental Dynamics 194 177-192. (doi:10.1002/aja.1001940303)

Rosen V 2006 BMP and BMP inhibitors in bone. Annals of the New York Academy of Sciences 1068 19-25. (doi:10.1196/annals.1346.005)

Segditsas S, Sieber O, Deheragoda M, East P, Rowan A, Jeffery R, Nye E, Clark S, Spencer-Dene B, Stamp G et al. 2008 Putative direct and indirect Wnt targets identified through consistent gene expression changes in APC-mutant intestinal adenomas from humans and mice. Human Molecular Genetics 17 3864-3875. (doi:10.1093/hmg/ ddn286)

Shima JE, McLean DJ, McCarrey JR \& Griswold MD 2004 The murine testicular transcriptome: characterizing gene expression in the testis during the progression of spermatogenesis. Biology of Reproduction $\mathbf{7 1}$ 319-330. (doi:10.1095/biolreprod.103.026880)
Skinner MK 2005 Regulation of primordial follicle assembly and development. Human Reproduction Update 11 461-471. (doi:10. 1093/humupd/dmi020)

Sudo S, Avsian-Kretchmer O, Wang LS \& Hsueh AJ 2004 Protein related to DAN and cerberus is a bone morphogenetic protein antagonist that participates in ovarian paracrine regulation. Journal of Biological Chemistry 279 23134-23141. (doi:10.1074/jbc.M402376200)

Received 3 January 2014

First decision 27 January 2014

Revised manuscript received 19 February 2014

Accepted 10 March 2014 